\title{
Searching for planetary-mass T-dwarfs in the core of Serpens
}

\author{
L. Spezzi ${ }^{1}$, C. Alves de Oliveira ${ }^{2}$, E. Moraux ${ }^{3}$, J. Bouvier ${ }^{3}$, E. Winston ${ }^{4}$, P. Hudelot ${ }^{5}$, H. Bouy ${ }^{6}$, and J.-C. Cuillandre ${ }^{7}$ \\ 1 European Southern Observatory, Karl-Schwarzschild-Strasse 2, 85748 Garching bei München, Germany \\ e-mail: 1spezzi@eso.org \\ 2 Herschel Science Centre, European Space Astronomy Centre (ESA), PO Box, 78, 28691 Villanueva de la Cañada, Madrid, Spain \\ 3 UJF-Grenoble 1/CNRS-INSU, Institut de Planétologie et d'Astrophysique de Grenoble (IPAG) UMR 5274, 38041 Grenoble, France \\ ${ }^{4}$ European Space Agency (ESTEC), PO Box 299, 2200 AG Noordwijk, The Netherlands \\ 5 Institut d'Astrophysique de Paris, UMR 7095 CNRS, Université Pierre et Marie Curie, 98bis boulevard Arago, 75014 Paris, France \\ ${ }^{6}$ Centro de Astrobiología (INTA-CSIC), LAEFF, PO Box 78, 28691 Villanueva de la Cañada, Spain \\ 7 Canada-France-Hawaii Telescope, 65-1238 Mamalahoa Hwy, Kamuela, HI 96743, USA
}

Received 8 May 2012 / Accepted 1 August 2012

\begin{abstract}
Context. The knowledge of the present-day mass function of young clusters and the mass of their coolest substellar members is essential to clarify the brown dwarf formation mechanism, which still remains a matter of debate.

Aims. We searched for isolated planetary-mass T-dwarfs in the $\sim 3$ Myr old Serpens Core cluster.

Methods. We performed a deep imaging survey of the central part of this cluster using the WIRCam camera at the CFHT. Observations were performed through the narrow-band $\mathrm{CH}_{4}$ off and $\mathrm{CH}_{4}$ on filters, to identify young T-dwarfs from their $1.6 \mu$ m methane absorption bands, and the broad-band $J H K_{\mathrm{S}}$ filters, to better characterize the selected candidates. We complemented our WIRCam photometry with optical imaging data from MegaCam at CFHT and Suprime-Cam at the Subaru telescope and mid-infrared flux measurements from the Spitzer "core to disk" (c2d) Legacy Survey.

Results. We report four faint T-dwarf candidates in the direction of the Serpens Core with $\mathrm{CH}_{4} \mathrm{On}-\mathrm{CH}_{4}$ off above 0.2 mag, estimated visual extinction in the range 1-9 mag and spectral type in the range T1-T5 based on their dereddened $\mathrm{CH}_{4} \mathrm{On}-\mathrm{CH}_{4}$ off colors. Comparisons with T-dwarf spectral models and optical to mid-infrared color-color and color-magnitude diagrams, indicate that two of our candidates (ID 1 and 2) are background contaminants (most likely heavily reddened low-redshift quasars). The properties of the other two candidates (ID 3 and 4) are consistent with them being young members of the Serpens Core cluster, although our analysis can not be considered conclusive. In particular, ID 3 may also be a foreground T-dwarf. It is detected by the Spitzer c2d survey but only flux upper limits are available above $5.8 \mu \mathrm{m}$ and, hence, we can not assess the presence of a possible disk around this object. However, it presents some similarities with other young T-dwarf candidates (S Ori 70 in the $\sigma$ Orionis cluster and CFHT_J0344+3206 in the direction of IC 348). If ID 3 and 4 belong to Serpens, they would have a mass of a few Jupiter masses and would be amongst the youngest, lowest mass objects detected in a star-forming region so far.
\end{abstract}

Key words. stars: formation - brown dwarfs - ISM: clouds - ISM: individual objects: Serpens Core - stars: low-mass

\section{Introduction}

Understanding the brown dwarf (BD) formation mechanism is a key point to assess what determines and which is the minimum mass for star formation. Although two decades have passed since the first unambiguous observations of BDs (Rebolo et al. 1995; Nakajima et al. 1995), this issue is still under debate. According to the star formation theory, the minimum mass for star formation is set by the so called mass fragmentation limit, i.e. the mass at which one object cannot contract further because the radiated heat prevents it to collapse further. This limit is of the order of a 5-10 $M_{\mathrm{J}}$ (Low \& Lynden-Bell 1976; Rees 1976) and may be lower $\left(\sim 1 M_{\mathrm{J}}\right)$ in the presence of magnetic fields (Boss 2001; Boyd \& Whitworth 2005). Observationally, several young star forming regions have been probed to faint magnitudes at both optical and near-infrared wavelengths to look for a possible cut-off at the low-mass end of the mass function and the results significantly vary from region to region. A possible cutoff below $\sim 6 M_{\mathrm{J}}$ up to $20 M_{\mathrm{J}}$ have been reported in NGC 1333 (e.g., Scholz et al. 2009, 2012). Moreover, there is no universal agreement on the behavior of the mass function over this cut-off. A search for T-dwarfs in IC 348 by Burgess et al. (2009) suggests that an extrapolation of the log-normal mass function (Chabrier 2003) may hold in the substellar and planetary-mass regimes. A similar study in the Upper Sco association favors a turn-down in the mass function below $10 M_{\mathrm{J}}$ (Lodieu et al. 2011). In the Pleiades Casewell et al. (2007) reported several L/T-type candidates with masses as low as $10 M_{\mathrm{J}}$, implying that the slope of the mass function in this mass regime agrees, within the uncertainties, with the values inferred from earlier studies at higher masses (Dobbie et al. 2002; Moraux et al. 2003; Lodieu et al. 2007; Casewell et al. 2007). Finally, Kirkpatrick et al. (2012) find tantalizing hints that the number of BDs in the field continues to rise from late-T to early-Y.

To address these questions, a large observing key-program (P.I. J. Bouvier) was conducted at the Canada France Hawaii Telescope (CFHT) aimed at characterizing the stellar population of nearby star forming regions using the Wide Field IR Camera (WIRCam). One of the main objectives of this program is to look for the lowest mass members of these clusters and investigate the cut-off at the low mass end of the mass function, using a technique based on narrow-band methane imaging (e.g., Burgess et al. 2009). 
In this paper, we report the results of this observing program in the Serpens Core cluster, specifically focusing on the methane filters imaging to search for young T-dwarfs candidates. The Serpens Core region is an example of a very young, deeply embedded $\left(A_{\mathrm{V}} \approx 40 \mathrm{mag}\right)$ cluster, containing a high percentage of protostars (Davis et al. 1999; Testi et al. 2000; Kaas et al. 2004; Eiroa et al. 2006; Harvey et al. 2007; Winston et al. 2007). With an age between 2 and $~ 6 \mathrm{Myr}$ (Winston et al. 2009; Oliveira et al. 2009) and a distance estimated between $260 \pm 37$ pc (Straižys et al. 2003) and $415 \pm 5$ pc (Dzib et al. 2010), the Serpens cloud core is one of the nearest regions of clustered star formation to the Sun. Therefore, it is an excellent candidate for study as it is close enough to both resolve the individual members and detect the lowest mass members to below the hydrogen-burning limit. Throughout this paper, we assume a typical age of $3 \mathrm{Myr}$ (Winston et al. 2009) for the Serpens Core cluster and the minimum distance of $260 \pm 37$ pc (Straižys et al. 2003) ${ }^{1}$. The region is also particularly interesting for BD studies because only a few dedicated searches have been reported in the literature. The first young BD in Serpens was discovered by Lodieu et al. (2002) and the cluster substellar population count so far about 3 spectroscopically confirmed BDs (Lodieu et al. 2002; Shirono et al. 2011) and 40 BD candidates, a considerable fraction of which still surrounded by prominent accretion disks (Eiroa et al. 2006).

Canonical approaches on BD studies and related issues (see, e.g., Jayawardhana et al. 2003) rely on the identification and spectral classification of BDs. However, optical/near-infrared (IR) spectroscopy (see, e.g., McLean et al. 2003; Geballe et al. 2002) has proved impossible for very faint distant BDs with current telescopes and very time-consuming for large BD samples. To overcome these limitations, new BD classification methods have been devised on the basis of near-IR broad-band (see, e.g., Leggett et al. 2010; Schmidt et al. 2010, and references therein) and narrow-band imaging that targets unique molecular features of $\mathrm{L}$ and $\mathrm{T}$ dwarfs, in particular, water and methane bands (see, e.g., Gorlova et al. 2003; Mainzer et al. 2004; Spezzi et al. 2011). These techniques are equivalent to extremely lowresolution spectroscopy and can be confidently applied for statistical purposes, e.g., to detect and classify bona fide BDs in large imaging surveys. Our search for T-dwarfs in Serpens is based on narrow-band methane $\left(\mathrm{CH}_{4}\right)$ imaging. We target, in particular, the $\mathrm{CH}_{4}$ absorption band centered at $1.66 \mu \mathrm{m}$, which forms in the atmosphere of objects cooler than $T_{\text {eff }} \lesssim 1200 \mathrm{~K}$ (defyning the L/T dwarf boundary; Burrows et al. 2006). This technique has been successfully applied to a search for T-dwarfs in the young IC 348 cluster (Burgess et al. 2009).

This paper is organized as follows. Section 2 describes the WIRCam observations in the Serpens Core and the catalog extraction procedure. In Sects. 3, 4 we use this catalog to identify possible T-dwarfs on the basis of methane absorption bands and estimate their intrinsic properties ( $T_{\mathrm{eff}}, A_{\mathrm{V}}$, etc.). In Sect. 5 we investigate the nature of our T-dwarfs candidates using complementary near to mid-IR data and T-dwarf spectral models. The conclusions of this work are given in Sect. 6.

\section{Observations and data reduction}

The imaging observations presented in this work were obtained as part of a large CFHT key program (08AF98) aimed

\footnotetext{
1 This choice is appropriate because we use the distance to Serpens to estimate the lower limit to the interstellar extinction towards the cloud (see also Sect. 4).
}

Table 1. Journal of the observations.

\begin{tabular}{lcccc}
\hline \hline Field & $\begin{array}{c}\text { Date } \\
(\mathrm{d} / \mathrm{m} / \mathrm{y})\end{array}$ & Filter & $\begin{array}{c}T_{\exp } \\
(\mathrm{s})\end{array}$ & $\begin{array}{c}\text { Seeing } \\
\left({ }^{\prime \prime}\right)\end{array}$ \\
\hline Serpens Core & $17-19 / 05 / 2008$ & $\mathrm{CH}_{4}$ off & 2520 & 0.6 \\
& $\begin{array}{c}17-19 / 05 / 2008 \\
\mathrm{CH}_{4} \text { on }\end{array}$ & 7560 & 0.6 \\
& $20 / 04 / 2008$ & $J$ & 8405 & 0.7 \\
& $20 / 04 / 2008$ & $H$ & 1405 & 0.7 \\
& $20 / 04 / 2008$ & $K_{\mathrm{S}}$ & 1405 & 0.7 \\
\hline
\end{tabular}

at the characterisation of the low-mass population of several young star-forming regions (P.I. J. Bouvier). The data were obtained in queue-scheduled observing mode between 20 April and 19 May 2008 using the WIRCam at CFHT. The WIRCam consists of four Hawaii-II2-RG $2048 \times 2048$ array detectors with a pixel scale of $0.3^{\prime \prime}$ or $0.15^{\prime \prime}$ with microdithering (Puget et al. 2004). The four detectors are arranged in a $2 \times 2$ pattern, with a total field of view of $21.5^{\prime} \times 21.5^{\prime}$ and small gaps of $45^{\prime \prime}$ between adjacent chips. The average $\mathrm{CCD}$ read-out noise and gain are 30 e- and 3.8 e-/ADU, respectively.

In this paper, we focus on the observations of the Serpens Core cluster obtained through the $\mathrm{CH}_{4}$ off $\left(\lambda_{\mathrm{C}}=1.58 \mu \mathrm{m}, \Delta \lambda=\right.$ $0.1 \mu \mathrm{m})$ and $\mathrm{CH}_{4}$ on $\left(\lambda_{\mathrm{C}}=1.69 \mu \mathrm{m}, \Delta \lambda=0.1 \mu \mathrm{m}\right)$ filters, along with observations through the $J H K_{\mathrm{S}}$ broad-band filters obtained using micro-dithering. A single WIRCam pointing centered at $\mathrm{RA}=18^{\mathrm{h}} 29^{\mathrm{m}} 57^{\mathrm{s}}$ and $\mathrm{Dec}=+01^{\mathrm{d}} 13^{\mathrm{m}} 08^{\mathrm{s}}$ was sufficient to cover the Serpens Core cluster. Each tile in each filter was observed using a 7-point dithering pattern selected to fill the gaps between detectors and accurately subtract the sky background. Exposure times, seeing and air mass conditions are summarized in Table 1.

Individual images were primarly processed using the standard CFHT Data Processing and Calibration Pipeline for WIRCam (I'iwi; Albert et al., in prep.), which includes bias subtraction, flat-fielding, non-linearity correction, cross-talk removal, sky subtraction, and astrometric calibration. The astrometric World Coordinate System (WCS) solution is purely linear (i.e., assumes a constant scale) and is determined in two steps. Knowing the geometry of the detectors (WIRCam has a field distortion of about $1 \%$ in the corner of the mosaic), a first fit using all 2MASS stars found on the whole mosaic is performed. Then, provided enough stars are found, the same fit method operates on each individual detectors to refine the WCS solution individually. This generally yields an astrometric accuracy of $\sim 1$ arcsec and the rms scatter of the resulting WCS solution is generally about $0.5 \operatorname{arcsec}^{2}$.

Afterwards, the combination of individual exposures into final stacked images and their photometric calibration were performed using the SCAMP and SWarp standard packages by Terapix, the data reduction centre at the Institut d'Astrophysique de Paris, France (see Marmo 2007, and references therein). The photometric calibration of the WIRCam data in the broad-band filters is done as part of the nominal pipeline reduction using 2MASS stars in the observed frames and images are renormalized to an arbitrary photometric zero-point of 30 mag. Typical estimated errors in the WIRCam zero point determination are of the order of $0.05 \mathrm{mag}$, depending on the number of $2 \mathrm{MASS}$ stars available, on the variability of those stars and on small problems in flat-fielding and/or sky subtraction from night to night.

2 For further details, we defer the reader to the WIRCam webpage: http://www . cfht . hawaii . edu/Instruments/Imaging/WIRCam/ IiwiVersion1Doc.html\#Part5. 
A further check/refinement of the extracted $J H K_{\mathrm{S}}$ magnitudes was performed using 2MASS photometry for the brightest stars in the field, as is described in the next section (i.e., Sect. 2.1). Images in the methane filters have no external photometric calibration and the $\mathrm{CH}_{4}$ off and $\mathrm{CH}_{4}$ on magnitudes are given here on an arbitrary albeit internally consistent scale, so that $\mathrm{H}-\mathrm{CH}_{4} \mathrm{On}=$ $\mathrm{H}-\mathrm{CH}_{4} \mathrm{off}=0$ for unreddened field dwarfs.

\subsection{Photometric catalog}

The source extraction and photometry were performed by using a combination of SExtractor (Bertin \& Arnouts 1996) and PSFex (Bertin 2011). SExtractor extracts well defined stellarlike objects, which are used by PSFex to compute a point spread function (PSF) model that is allowed to vary with position on the detector. Then, SExtractor uses this PSF model to accurately extract and measure the photometry of all the sources detected on the image.

T-dwarf candidates are expected to be fainter and, hence, possibly missed in the $\mathrm{CH}_{4}$ on image because of their $\mathrm{CH}_{4}$ absorption. Thus, we first perform the source extraction on the $\mathrm{CH}_{4}$ off image and then use this catalog as input list when running Sextractor on the $\mathrm{CH}_{4}$ on image. To ensure the detection of all the faint sources present in the images the extraction criteria used are not too stringent. An object is extracted if it complies with the requirement to have three contiguous pixels (DETECT_MINAREA = 3) with fluxes 1.5 times above the background variation (DETECT_THRESH = ANALYSIS_ THRESH $=1.5$ ). In order to minimize the number of spurious detections, the SExtractor cleaning efficiency parameter was set to the maximum value (CLEAN_PARAM = 1). An inspection of the images and detections showed that all the objects seen by eye are detected. However, this visual inspection showed that a significant fraction of blended sources was missed. A higher selection threshold (DETECT_THRESH = ANALYSIS_THRESH = 5) allowed us to recover those sources and we created a final master catalog combining the results of the two runs and containing $\sim 130000$ objects. The summary of saturation limits and limiting magnitudes, after cleaning for saturated objects and obvious spurious detections/artifacts (i.e. Sextractor FLAGS $\leq 2$ ), is given in Table 2. The approximate completeness limit in each filter was derived as the point where the histogram of the magnitudes not corrected for extinction (Fig. 1) diverge from the dotted line, which represents the linear fit to the logarithmic number of objects per magnitude bin, calculated over the intervals of better photometric accuracy (Santiago et al. 1996; Wainscoat et al. 1992).

About $60 \%$ of the objects detected in $\mathrm{CH}_{4}$ off and $\mathrm{CH}_{4}$ on images have a counterpart in the broad-band images. An analogous procedure was applied to obtain $J H K_{\mathrm{S}}$ photometry. We perform the source extraction on the $J, H$ and $K_{\mathrm{S}}$ images independently using Sextractor and adopting the same configuration parameters as for the methane filters, i.e. DETECT_MINAREA $=3$, DETECT_THRESH $=$ ANALYSIS_THRESH $=1.5$ and CLEAN_PARAM $=1$. A second run was also performed on the $J H K_{\mathrm{S}}$ images using an higher selection threshold (DETECT_THRESH $=$ ANALYSIS_THRESH $=5$ ) to recover the blended sources. The final master catalog, obtained by combining the results of the two runs, contains $\sim 94000$ sources for the $J$ filter, $\sim 81000$ for the $H$ filter and $\sim 87000$ for the $K_{\mathrm{S}}$ filter. Approximately 4000 of the brightest stars in our field have a counterpart in the 2MASS catalogue. We used this sample to check the photometric accuracy and pipeline reduction of the WIRCam images and to estimate the difference between the
Table 2. Saturation limit, limiting magnitudes at the $10 \sigma$ and $5 \sigma$ level and completeness limit in each filter.

\begin{tabular}{lcccc}
\hline \hline Filter & $\begin{array}{c}\text { Saturation } \\
\text { limit }\end{array}$ & $\mathrm{Mag}_{10 \sigma}$ & $\mathrm{Mag}_{5 \sigma}$ & $\begin{array}{c}\text { Completeness } \\
\text { limit }\end{array}$ \\
\hline $\mathrm{CH}_{4}$ off & 13.0 & 20.1 & 20.9 & 19.5 \\
$\mathrm{CH}_{4}$ on & 13.5 & 20.6 & 21.3 & 19.5 \\
$J$ & 13.5 & 20.8 & 21.5 & 20.5 \\
$H$ & 12.5 & 19.0 & 19.8 & 19.0 \\
$K_{\mathrm{S}}$ & 12.5 & 18.6 & 19.3 & 18.5 \\
\hline
\end{tabular}

CFHT and 2MASS photometric systems. The mean magnitude differences between the two systems is found to be $0.07,0.06$, and $0.1 \mathrm{mag}$ for $J, H$, and $K_{\mathrm{S}}$, respectively, which is of the order of expected zero point uncertainty ( $\sim 0.05$, Sect. 2$)$. The dispersion of the differences is $\sim 0.08$ mag form the three filters and reflects the possible sources of error mentioned above (i.e., number and variability of the available 2MASS stars, nonoptimal flat-fielding and/or sky subtraction from night to night, etc.; Sect. 2). Given that the dispersion is as large as the typical magnitude difference between the two systems, we choose to not correct our photometry for these offsets. Moreover, this comparison hold for the brightest stars in our images, because fainter ones are undetected in the 2MASS survey. This implies that color effects arising from CFHT filters can not be taken into account in this calibration, although they are expected to be significant because the WIRCam and 2MASS filters design differ substantially. Thus, throughout this work, all the WIRCam $J, H$, and $K_{\mathrm{S}}$ photometry is given in the CFHT Vega system. The saturation limits and limiting magnitudes in the broad-band filters, after cleaning for saturated objects and obvious spurious detections/artifacts (i.e., Sextractor FLAGS $\leq 2$ ), are given in Table 2. The approximate completeness limit in each broad-band filter was derived as for the methane filters (Fig. 1).

The catalogues from all filters are combined into a single database by requiring a positional match better than $1^{\prime \prime}$, corresponding to the astrometric accuracy of our images (see Sect. 2).

\section{T-dwarf candidate selection}

As demonstrated by Burgess et al. (2009), the flux ratio between the $\mathrm{CH}_{4}$ on and $\mathrm{CH} 4$ off filters gives a good measure of the strength of the methane absorption in T-dwarfs. Specifically, the $\mathrm{CH}_{4}$ on filter probes the $\mathrm{CH}_{4}$ absorption band centered at $\sim 1.66 \mu \mathrm{m}$ and typical of T-dwarfs, while the $\mathrm{CH}_{4}$ off filter covers a wavelength range relatively featureless in T-type objects and, hence, can be used as the primary continuum filter.

As shown by Burgess et al. (2009) in their Fig. 2, the $\mathrm{CH}_{4}$ on$\mathrm{CH}_{4}$ off color in the CFHT methane filters is roughly equal to zero for L-type and earlier type dwarfs and smoothly increases towards later spectral type, so that T-dwarfs have colors above $\sim 0.1$. Fig. 2 shows the $\mathrm{CH}_{4}$ on- $\mathrm{CH}_{4}$ off against $\mathrm{CH}_{4}$ off diagram for point-like objects detected in the observed area in Serpens Core. We use this diagram to select T-dwarf candidates following the steps described below:

- Most of the stars in our field have spectral type earlier than $\mathrm{L}$, as demonstrate by the over-density of points at $\mathrm{CH}_{4}$ on$\mathrm{CH}_{4}$ off $\approx 0$ in Fig. 2. We use the median $\mathrm{CH}_{4}$ on- $\mathrm{CH}_{4}$ off color of these stars as a function of the $\mathrm{CH}_{4}$ off magnitude to define the reference template with respect to which the $\mathrm{CH}_{4}$ absorption feature is sought. The continuous line and 

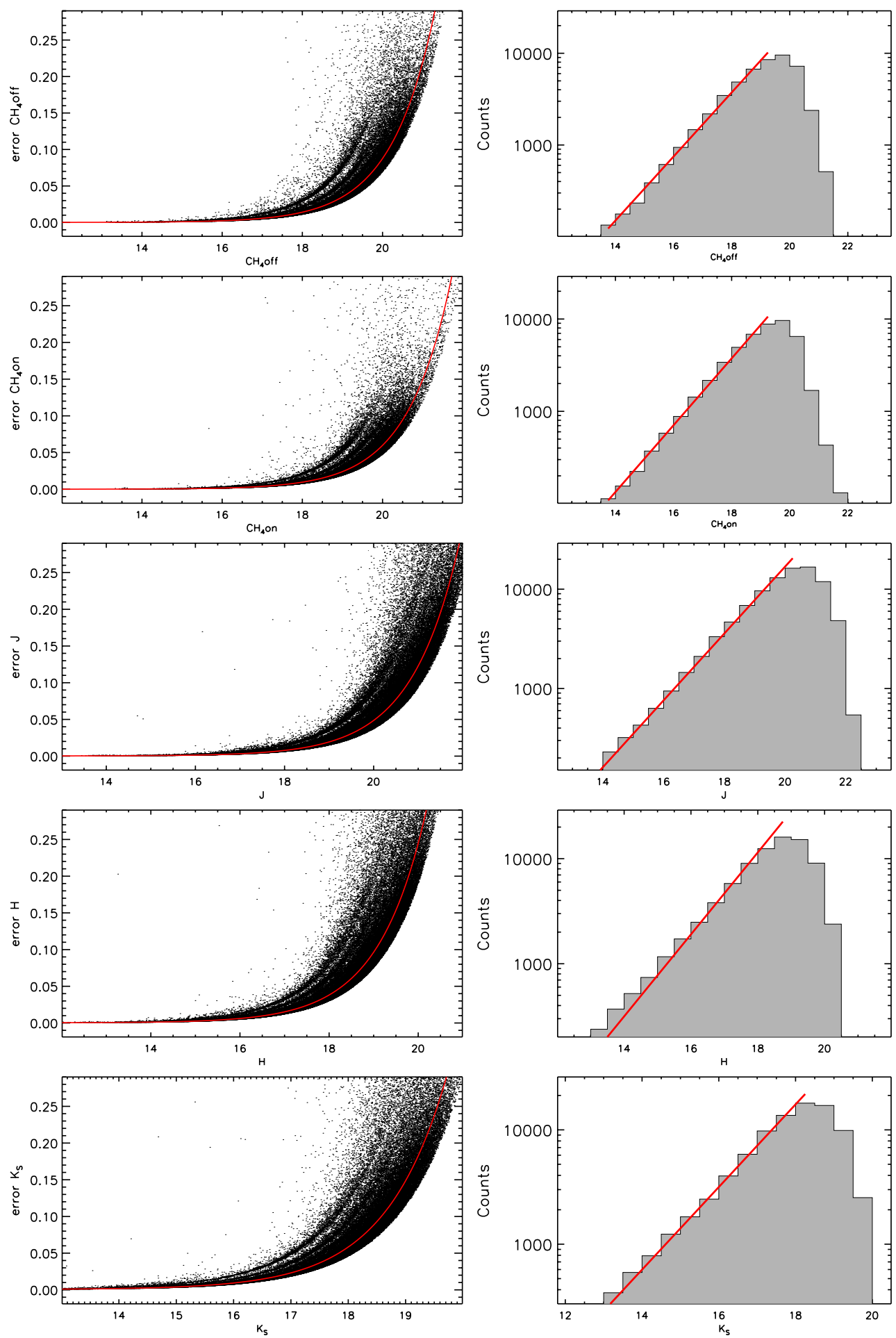

Fig. 1. Left panels: photometric errors as a function of PSF magnitudes and relative exponential fit (continuous line) for all the point-like sources detected in the $\mathrm{CH}_{4} \mathrm{off}, \mathrm{CH}_{4}$ on and $\mathrm{JHK}_{\mathrm{S}}$ images. Right panels: number of detection as a function of magnitude. The line shows the linear fit to the histogram points used to find the turning point of the distribution, indicating our completeness limit.

the line-filled area in Fig. 2 show the median locus of field stars and their dispersion $(\sigma)$, respectively.

- We consider the photometric uncertainty on the $\mathrm{CH}_{4}$ on$\mathrm{CH}_{4} \mathrm{Off}$ color $\left(\delta=\sqrt{\Delta \mathrm{CH}_{4} \mathrm{On}^{2}+\Delta \mathrm{CH}_{4} \mathrm{Off}^{2}}\right)$ and select a first sample of T-dwarf candidates by considering all those objects with $\left(\mathrm{CH}_{4}\right.$ on- $\mathrm{CH}_{4}$ off $)-\delta$ color exceeding that of the median locus of field stars by more than $3 \sigma$. The objects just below this limit could still be early T-dwarfs but it would be more difficult to extract them because of photometric errors. With this criterion $\sim 200$ sources were selected.

- This sample was visually inspected on both the $\mathrm{CH}_{4}$ off and $\mathrm{CH}_{4}$ on images and the $J H K_{\mathrm{S}}$ images. Most of the objects turned out to be nebulous detections, ghosts or detector cross-talk, spurious detections/artifacts in the vicinity of saturated objects or image edges. Thus, the initial sample was reduced to a shortlist of only 4 point-like candidates. 
Table 3. Photometry of the T-dwarf candidates.

\begin{tabular}{cccccccccc}
\hline \hline ID & $\begin{array}{c}\text { RA J2000 } \\
\text { (hh:mm:ss })\end{array}$ & $\begin{array}{c}\text { Dec J2000 } \\
(\mathrm{dd}: \mathrm{mm}: \mathrm{ss})\end{array}$ & $r_{\text {CFHT }}$ & $i_{\text {Subaru }}$ & $\mathrm{CH}_{4}$ off & $\mathrm{CH}_{4}$ on & $J$ & $H$ & $K_{\mathrm{S}}$ \\
\hline 1 & $18: 29: 56.58$ & $+01: 18: 35.93$ & - & $22.98 \pm 0.03$ & $17.77 \pm 0.05$ & $18.07 \pm 0.05$ & $19.10 \pm 0.05$ & $17.71 \pm 0.06$ & $16.99 \pm 0.05$ \\
2 & $18: 29: 57.64$ & $+01: 19: 40.61$ & - & $22.67 \pm 0.03$ & $17.61 \pm 0.05$ & $18.10 \pm 0.05$ & $18.84 \pm 0.05$ & $17.64 \pm 0.06$ & $17.07 \pm 0.06$ \\
3 & $18: 30: 27.89$ & $+01: 14: 52.22$ & - & $23.98 \pm 0.10$ & $18.75 \pm 0.05$ & $19.16 \pm 0.06$ & $19.38 \pm 0.06$ & $19.08 \pm 0.16$ & $18.90 \pm 0.27$ \\
4 & $18: 30: 37.24$ & $+01: 18: 37.68$ & $23.57 \pm 0.07$ & $22.14 \pm 0.02$ & $18.61 \pm 0.06$ & $18.84 \pm 0.06$ & $19.71 \pm 0.07$ & $18.67 \pm 0.09$ & $18.25 \pm 0.10$ \\
\hline
\end{tabular}

Notes. $\mathrm{CH}_{4}$ on and $\mathrm{CH}_{4}$ off magnitudes are in the WIRCam system, while riJHK photometry is given in the Vega system.

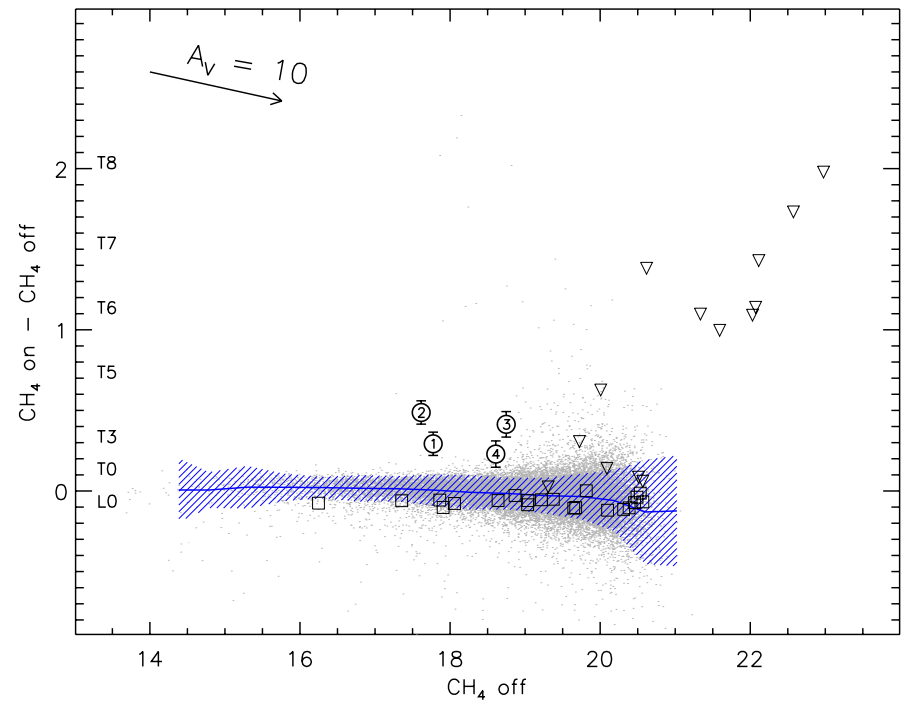

Fig. 2. $\mathrm{CH}_{4}$ on- $\mathrm{CH}_{4}$ off vs. $\mathrm{CH}_{4}$ off diagram for the point-like objects detected in Serpens Core (small grey dots). The continuous line and the line-filled area show the median locus of field stars and their dispersion, respectively. The squares and triangles show the location of confirmed field L and T dwarfs, respectively; their magnitudes have been shifted to the distance modulus of Serpens. The circles show the position of our the T-dwarf candidates; their ID number (see Tables 3, 4) is indicated and errors bars are within the circle size. The extinction vector is shown for $A_{\mathrm{V}}=10$ mag. The $\mathrm{CH}_{4}$ on- $\mathrm{CH}_{4}$ off vs. spectral type calibration relation by Burgess et al. (2009) is also reported on the $y$-axis.

The PSF photometry of these four T-dwarf candidates in the $\mathrm{CH}_{4} \mathrm{on} / \mathrm{CH}_{4}$ off filters and in $J H K_{\mathrm{S}}$ is listed in Table 3. Thumbsized images of the four candidates are shown in Fig. 3.

\section{Reddening and spectral type estimates}

Knowledge of the candidates' extinction $\left(A_{\mathrm{V}}\right)$ is required in order to be able to estimate their spectral type and collect further information on their nature.

We do not know a priori whether our candidates are field T-dwarfs or young members of Serpens. In both cases, we cannot give accurate $A_{\mathrm{V}}$ value for each object based only on photometry, because we do not know their distance; they could be foreground objects closer than Serpens, cloud members located between 260 and 415 pc, i.e. the distance range of Serpens (see Table 2 by Dzib et al. 2010) or even being located beyond the cloud. Thus, we estimate the range of possible extinction values for each candidate as follows:

- According to the study by Chapman et al. (2009), the midinfrared extinction law in Serpens is consistent with the
Weingartner \& Draine (2001) $R_{\mathrm{V}}=3.1$ diffuse interstellarmedium dust model below $A_{K} \lesssim 1\left(A_{\mathrm{V}} \lesssim 9\right)$. We use this prescription to define the direction of the extinction vector.

- Assuming that our candidates belong to Serpens, extinction can be estimated using color-color diagrams of $\mathrm{CH}_{4}$ on$\mathrm{CH}_{4}$ off versus $J-H, J-K_{\mathrm{S}}$ and $H-K_{\mathrm{S}}$, as plotted in Fig. 4. The extinction is computed for each candidate using the extinction vector and regressing the objects back towards the 3 Myr PMS isochrone (Chabrier et al. 2000), i.e. the typical age of the Serpens cloud core population (Winston et al. 2009; Oliveira et al. 2009). The extinction values derived through this method $\left(A_{\mathrm{V}}^{3 \mathrm{My}}\right)$ are reported in Table 4.

- Gutermuth (priv. comm.) calculated an extinction map of the Serpens Core using 2MASS data and the method explained in Gutermuth et al. (2007). This map has a spatial resolution of $25^{\prime \prime} \times 25^{\prime \prime}$ and entirely covers the area observed by us. By comparing this map with our WIRCam images, we estimate that the crowding of our field is such that typically $\sim 50$ stars fall in the map resolution box. This number is suitable for a statistically significant estimate of $A_{\mathrm{V}}$ in the given box/direction based on the Gutermuth et al. (2007) method. Because the extinction map takes into account all the extinction occurring along the line of sight, it can be used to estimate the maximum extinction towards our candidates under the assumption that they are located beyond the Serpens cloud. Moreover, the extinction assigned to each object ( $A_{\mathrm{V}}^{\text {map }}$ in Table 4 ) is the average value in a $25^{\prime \prime}$ box around the object itself and, as such, can be inaccurate for very young and highly embedded objects, which present peculiar extinction due to the material in their envelope/disk. In Sect. 5.2 we will see that this is not likely to be the case for our candidates. However, the average $A_{\mathrm{V}}$ value computed in a given $25^{\prime \prime}$ box can be still inaccurate for an object located in a specific position within the box, because of the effect of small scale differential reddening, presence of cloud filaments, etc.

- Finally, to estimate the lower limit to the extinction of our candidates, we assumed that they are foreground T-dwarfs located within $260 \mathrm{pc}$ of our Sun, i.e. the minimum distance estimated for Serpens. The typical diffuse absorption is taken to be $0.7-1 \mathrm{mag} / \mathrm{kpc}$ in the Solar Neighborhood (see, e.g., Ichikawa et al. 1982). Thus, interloping field objects between our Sun and Serpens would be subject to a $0 \lesssim A_{\mathrm{V}} \lesssim 0.2 \mathrm{mag}$ extinction.

For each candidate, we considered a minimum extinction of $0 \lesssim A_{\mathrm{V}} \lesssim 0.2 \mathrm{mag}$, the extinction occurring along the line of sight, given by the extinction map, and the extinction expected if the object belongs to the young population of Serpens $\left(A_{\mathrm{V}}^{\text {map }}\right.$ and $A_{\mathrm{V}}^{3 \mathrm{My}}$ in Table 4 , respectively) and calculated the relative dereddened $\mathrm{CH}_{4}$ on- $\mathrm{CH}_{4}$ off colors. We then used the $\mathrm{CH}_{4}$ on$\mathrm{CH}_{4}$ off vs. spectral type calibration by Burgess et al. (2009) (see 


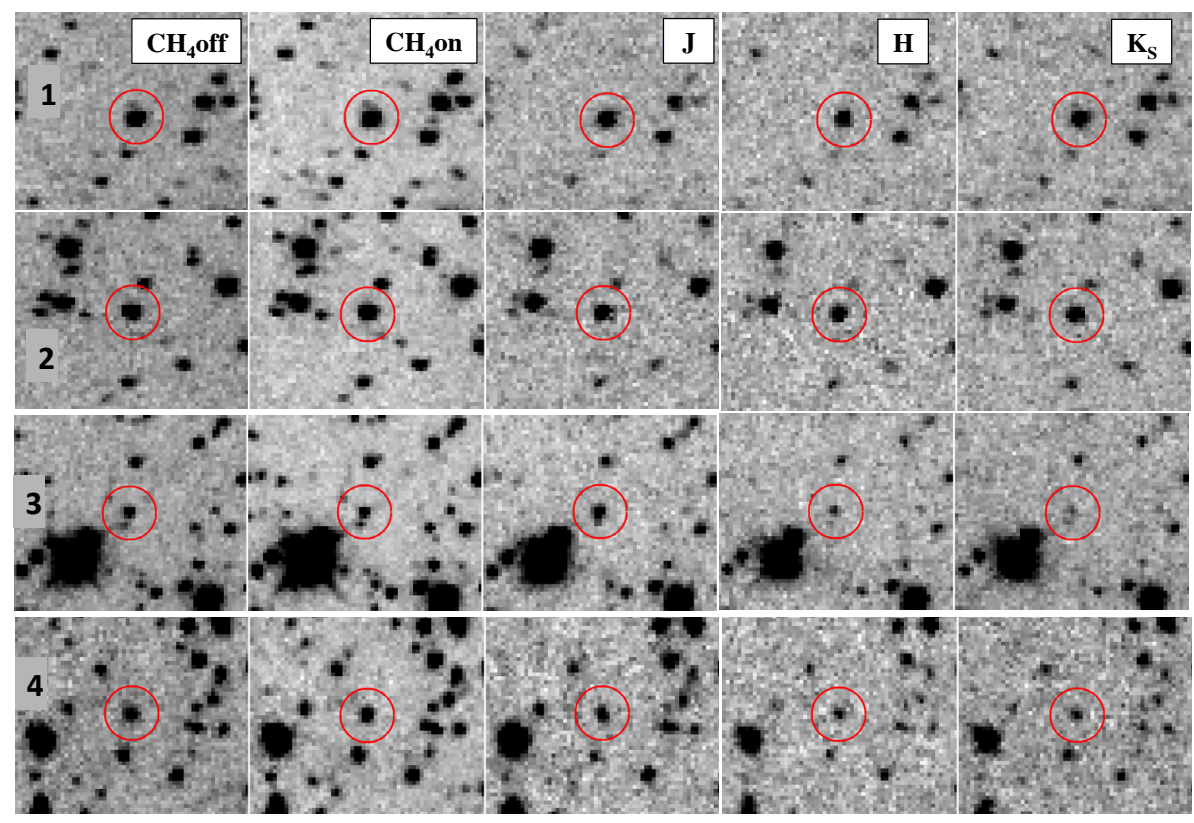

Fig. 3. Images of the four T-dwarfs candidates in each of the WIRCam@CFHT filter used in this work, as indicated in the labels. Each snapshot covers an area of $18^{\prime \prime} \times 15^{\prime \prime}$; north is up and east to the left.

Table 4. Estimates of visual extinction, spectral type, and effective temperature for our T-dwarf candidates based on their dereddened $\mathrm{CH}_{4}$ on $-\mathrm{CH}_{4}$ off colors.

\begin{tabular}{ccccc}
\hline \hline ID & $\begin{array}{c}A_{\mathrm{V}}^{\text {map }} \\
(\mathrm{mag})\end{array}$ & $\begin{array}{c}A_{\mathrm{V}}^{3 \text { My }} \\
(\mathrm{mag})\end{array}$ & Spec. type & $\begin{array}{c}T_{\text {eff }} \\
(\mathrm{K})\end{array}$ \\
\hline 1 & 9.2 & 9.4 & T2-T4 & $1390-1290$ \\
2 & 7.9 & 7.1 & T4-T5 & $1290-1190$ \\
3 & 4.2 & 1.0 & T3-T4 & $1360-1290$ \\
4 & 2.8 & 5.5 & T1-T3 & $1390-1360$ \\
\hline
\end{tabular}

Notes. $A_{\mathrm{V}}^{3 \mathrm{My}}$ is the visual extinction when assuming that the object belongs to the 3 My old population of Serpens, while $A_{\mathrm{v}}^{\text {map }}$ is derived from the extinction map by Gutermuth (priv. comm.).

their Fig. 2) to estimate the spectral type range consistent with these dereddened colors. Note that the calibration relation by Burgess et al. (2009) is based on field dwarfs; the actual colors of our LT dwarfs might be slightly different because of the effects that reduced gravity has on the opacities (see, e.g., Casewell et al. 2011, and references therein).

The spectral type estimates for our candidates are reported in Table 4, together with the corresponding effective temperature range according to the calibration relation by Vrba et al. (2004).

\section{On the nature of the T-dwarf candidates: are they young members of Serpens?}

In this section we use color-magnitude diagrams (CMDs), color-color (CC) diagrams and spectral energy distributions (SEDs) to assess whether the optical/IR properties of our T-dwarf candidates are consistent with those of field objects or indicate the presence of IR excess emission typical of very young objects, which would in turn indicate membership to the Serpens Core cluster. This analysis is performed using our
WIRCam data in combination with $r$ and $i$-band imaging data from MegaCam at CFHT and Suprime-Cam at the Subaru telescope, and Spitzer imaging.

\subsection{WIRCam near-IR color-magnitude and color-color diagrams}

Figure 5 shows the position of our candidates on the $J$ vs. $J-K_{\mathrm{S}}$ $\mathrm{CMD}$ and the $J-H$ vs. $H-K_{\mathrm{S}} \mathrm{CC}$ diagram before and after correction for interstellar reddening. In order to draw the dereddening vector for our candidates, we adopt the maximum extinction expected towards our candidates (i.e., the maximum between the $A_{\mathrm{V}}^{\text {map }}$ and $A_{\mathrm{V}}^{3 \mathrm{My}}$ value from Table 4); in this way, we display the maximum possible shift of their position in the CMD and $\mathrm{CC}$ diagram due to reddening effects.

We also plotted the 5 Gyr DUSTY and COND models and the confirmed field $\mathrm{L}$ and $\mathrm{T}$ dwarfs (Chiu et al. 2006; Golimowski et al. 2004; Knapp et al. 2004) to highlight the position expected for the field dwarf sequence. The locus expected for the young members of Serpens is indicated by the 3 Myr DUSTY and COND models and the young stellar object (YSO) population confirmed by the Spitzer core to disk (c2d) survey (Winston et al. 2007; Harvey et al. 2007). Finally, we plot for comparison the faint mid-T type object S Ori 70 found by Zapatero Osorio et al. (2002) towards the direction of the young $\sigma$ Orionis cluster and the dereddened position of CFHT_J0344+3206, a mid-T dwarf candidate found by Burgess et al. (2009) in the direction of IC 348. The DUSTY and COND models and the positions of the field LT-dwarfs, S Ori 70 and CFHT_J0344+3206 are shifted to the minimum distance estimated for Serpens (i.e. 260 pc). Note that it is still unclear whether S Ori 70 is a field brown dwarf or a young planetary mass member of $\sigma$ Orionis; however, proper motion and the near- and mid-IR colors measurements by Zapatero Osorio et al. (2008) support its membership to the cluster, with an estimated mass in the interval $2-7 M_{\odot}$. 

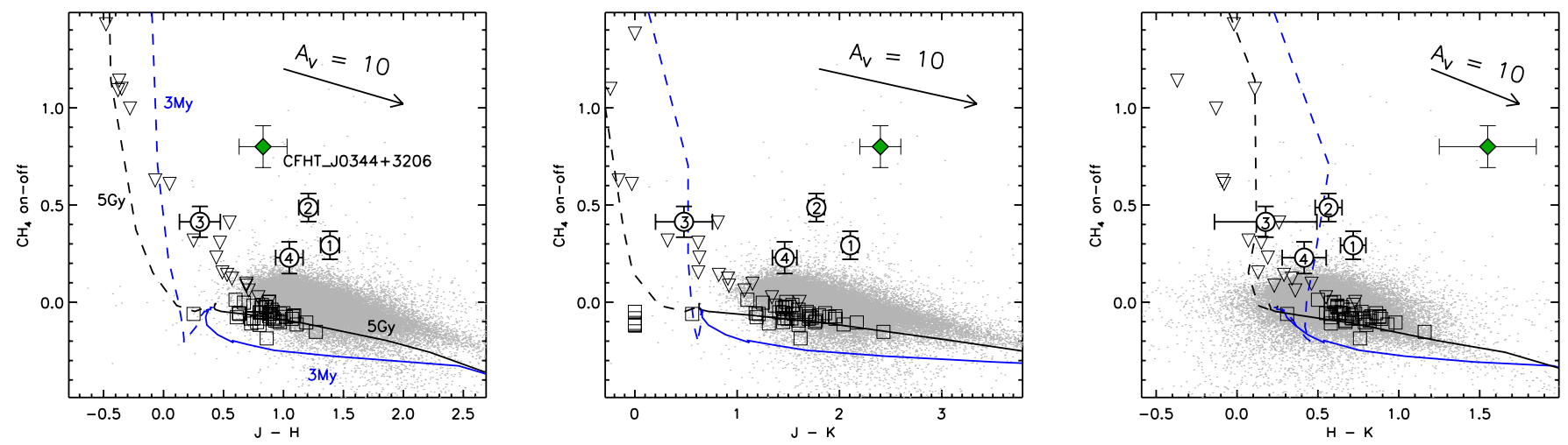

Fig. 4. $\mathrm{CH}_{4} \mathrm{On}-\mathrm{CH}_{4}$ off vs. $J-H, J-K$ and $H-K$ for the point-like objects detected in Serpens Core (small grey dots). The circles with error bars are the T-dwarf candidates in this study; their ID number is indicated. The squares and triangles show the location of confirmed field L and T dwarfs, respectively (Chiu et al. 2006; Golimowski et al. 2004; Knapp et al. 2004). The position of the CFHT_J0344+3206 mid-T dwarf candidate (green diamond) is plotted for comparison. The lines show the 5 Gyr and 3 Myr COND isochrones (dashed lines) and the 5 Gyr and 3 My DUSTY isochrones (continuous line), as indicated in the labels. The extinction vector is shown for $A_{\mathrm{V}}=10$ mag.

The position in both diagrams of two of our candidates, namely ID 1 and 2, is consistent with them being mid-T dwarfs. However, their dereddened magnitudes are brighter than expected for young T-dwarfs at the distance of Serpens and, hence, they might be field objects. They suffer from significant interstellar extinction (Table 4) and therefore can not be foreground objects. As discussed by Delorme et al. (2008), the only background contaminants may eventually be high redshift quasistellar objects (QSO; $z \gtrsim 6$ ) or heavily reddened low redshift QSOs, which appear point-like and share the same very red colors as cool dwarfs. Moreover, high redshift/heavily veiled QSOs can present a moderate methane-like absorption signal in the $\mathrm{CH}_{4}$ on- $\mathrm{CH}_{4}$ off difference due to the combinations of specific redshifts and emission lines (Richards et al. 2003). In Sect. 5.2 we use additional imaging in the $i$ and Spitzer pass-bands to assess whether this is the case for ID 1 and 2 .

ID 3 does not present a significant interstellar extinction and its near-IR colors are consistent with it being a field mid-T dwarf. Its position on the $J$ vs. $J-K_{S}$ diagram and spectral type are very similar to those of the mid-T type dwarf S Ori 70 in the $\sigma$ Orionis cluster and the T-dwarf candidate CFHT_J0344+3206 in the direction of IC 348. However, it is not redder than the sequence defined by field T-type dwarfs in the $J-H$ vs. $H-K_{\mathrm{S}} \mathrm{CC}$ diagram, as young objects should be. Indeed, the $K$-band flux is sensitive to gravity and it is fainter for young objects, especially for late T-dwarfs, because of the strongly reduced gravity (Zapatero Osorio et al. 2008). Since it is brighter than field objects at the distance of Serpens (Fig. 5, left panel), ID 3 may be a foreground field T-dwarf.

The interpretation of the CMD and CC diagram for candidate ID 4 is controversial, because it lies in both diagrams in a "transition region" mainly populated by field dwarfs but where faint and/or highly veiled YSOs might still be found. It cannot be a foreground object as it is significantly extincted (Table 4) and has near-IR dereddened colors consistent with it being an early T-dwarf. It is brighter than field objects (Fig. 5, left panel), but this is still consistent with it being young (the larger radius of YSOs make them brighter than older objects with the same spectral type). Thus, ID 4 may be a cluster member.

It is worth noting that these very same conclusions can be drawn from the inspection of the CC diagrams involving colors in the $\mathrm{CH}_{4}$ filters, which we presented in Fig. 4.

\subsection{Complementary MegaCam, Suprime-Cam and Spitzer data}

The Serpens Core cluster was observed on 2010-06-11 in the $r$ and $i$ bands with MegaCam (Boulade et al. 2003) at CFHT and on 2008-06-05 in the $i$-band with Suprime-Cam (Miyazaki et al. 2002), the wide field camera located at the prime focus of the Subaru Telescope. For details about these observations and the relative data reduction and calibration, we defer the reader to Bouy et al. (in prep.). In Table 3 we report the ri photometry of our four T-dwarf candidates calibrated to the Vega system. Only one of our candidates (ID 4) was detected in both filters, while the remaining three objects (ID 1,2 and 3) were detected only in the $i$-band.

We also searched for possible mid- to far-IR flux measurements of our T-dwarf candidates in the AKARI (Yamauchi et al. 2011), WISE (Wright et al. 2010) and Spitzer databases. The AKARI and WISE all-sky surveys turn out to be too shallow for the detection of our T-dwarf candidates. However, three of them (ID 1, ID 2 and ID 3) have been detected in the deeper Spitzer c2d Survey, which mapped Serpens together with other five nearby molecular clouds, using both IRAC and MIPS, the two imaging cameras on Spitzer (Evans et al. 2009). ID 4, the faintest of our candidates, was not detected by the Spitzer c2d Survey. The IRAC and MIPS $24 \mu \mathrm{m}$ fluxes for ID 1, ID 2 and ID 3 are reported in Table 5. We also computed for these three candidates the SED slope between the $K$-band $(2.2 \mu \mathrm{m})$ and the MIPS band at $24 \mu \mathrm{m}\left(\alpha_{[K \& 24 \mu \mathrm{m}]}\right.$, Table 5), which is traditionally used to assess the IR class of YSOs (Lada \& Wilking 1984). The $\alpha_{[K \& 24 \mu \mathrm{m}]}$ we obtain are upper limits to the actual SED slopes, because only flux upper limits are available at $24 \mu \mathrm{m}$ for our objects.

In Fig. 6 (left panel) we compare the position of our candidates in the $K_{\mathrm{S}}-$ [3.6] vs. [3.6]-[4.5] CC diagram with the locus expected for field dwarfs (Patten et al. 2006), the sequence defined by YSOs belonging to Serpens (Harvey et al. 2007; Winston et al. 2007) and the position of S Ori 70 and CFHT_J0344+3206.

In Fig. 6 (right panel) we use the $i-J$ vs. $J-[3.6]$ CC diagram to compare the colors of our candidates with the locus of YSOs in Serpens, the position of S Ori 70 and the colors expected for background QSOs, which might contaminate our sample. As demonstrated by Bouy et al. (2009), very low mass YSOs (with or without mid-IR excess associated to the presence 
Table 5. Spitzer fluxes and SED slopes $\left(\alpha_{[K \& 24 \mu \mathrm{m}]}\right)$ for the three T-dwarf candidates in this study detected by the Spitzer c2d survey.

\begin{tabular}{lccccccc}
\hline \hline ID & c2d Name & $\alpha_{[K \& 24 \mu \mathrm{m}]}$ & $\begin{array}{c}\text { IRAC 3.6 } \mu \mathrm{m} \\
(\mathrm{mJy})\end{array}$ & $\begin{array}{c}\text { IRAC 4.5 } \mu \mathrm{m} \\
(\mathrm{mJy})\end{array}$ & $\begin{array}{c}\text { IRAC 5.8 } \mu \mathrm{m} \\
(\mathrm{mJy})\end{array}$ & $\begin{array}{c}\text { IRAC } 8 \mu \mathrm{m} \\
(\mathrm{mJy})\end{array}$ & $\begin{array}{c}\text { MIPS 24 } \mu \mathrm{m} \\
(\mathrm{mJy})\end{array}$ \\
\hline 1 & SSTc2dJ182956.6+011836 & $-0.88 \pm 0.02$ & $0.0869 \pm 0.0084$ & $0.0610 \pm 0.0065$ & $0.0930 \pm 0.0307$ & $0.0464 \pm 0.0405$ & $<0.1410$ \\
2 & SSTc2dJ182957.6+011941 & $-1.06 \pm 0.02$ & $0.0667 \pm 0.0084$ & $0.0476 \pm 0.0102$ & $0.0393 \pm 0.0403$ & $<0.0028$ & $<0.0836$ \\
3 & SSTc2dJ183027.9+011452 & $0.27 \pm 0.04$ & $0.0295 \pm 0.0078$ & $0.0121 \pm 0.0108$ & $<0.0060$ & $<0.0590$ & $<0.3930$ \\
\hline
\end{tabular}
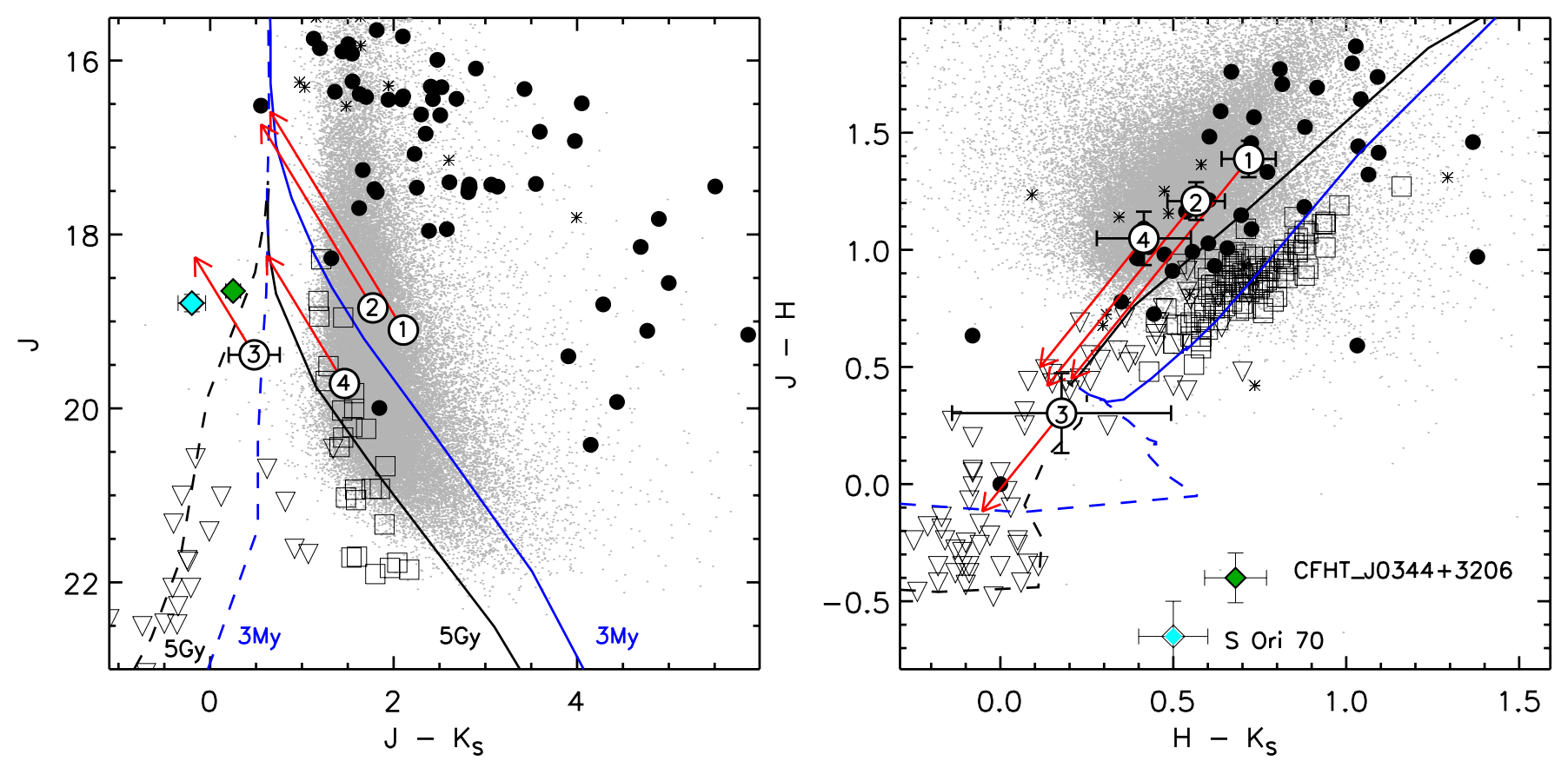

Fig. 5. $J$ vs. $J-K_{\mathrm{S}}$ (left panel) and $J-H$ vs. $H-K_{\mathrm{S}}$ diagrams (right panel) for the point-like objects detected in Serpens Core (small grey dots). The circles with error bars are the T-dwarf candidates in this study; their ID number is indicated and the arrows indicate their position after correction for interstellar reddening. The Serpens Core class II (black dots) and class III (asterisks) YSO population, the S Ori 70 mid-T dwarf (sky-blue diamond) and the CFHT_J0344+3206 mid-T dwarf candidate (green diamond) are plotted for comparison. The squares and triangles show the location of confirmed field L and T dwarfs (Chiu et al. 2006; Golimowski et al. 2004; Knapp et al. 2004), respectively, shifted to the distance of Serpens. Lines show the 5 Gyr and 3 My COND isochrones (dashed lines) and the 5 Gyr and 3 My DUSTY isochrones (continuous line).

of a circumstellar disk) and QSOs occupy two very distinct areas of this diagram, thus it can be used to effectively separated the two populations.

Finally, in Fig. 7 we present the SEDs of our four candidates from the optical up to $24 \mu \mathrm{m}$. The line-filled area in each SED plot represents the flux variation depending on the adopted reddening correction (see Sect. 4 and Table 4). The spectrum overplotted to each SED is the COND model spectrum by Allard et al. (2001) and Baraffe et al. (2003) with the same effective temperature as the object, normalized to the $J$-band flux; this model represents the pure stellar photospheric emission expected for an object of the given $T_{\text {eff }}$.

The Spitzer colors of ID 1 and ID 2 (Fig. 6) are in general agreement with those of class III objects with thin or no disks. This is also in agreement with the shape of their SEDs, which might present a small IR excess emission only at $24 \mu \mathrm{m}$ (Fig. 7). For both objects we find $\alpha_{[K \& 24 \mu \mathrm{m}]} \lesssim-1$, which is typical of class III objects with no prominent IR excess (Lada \& Wilking 1984; Greene et al. 1994). Thus, the overall Spitzer colors/SED for these two objects indicate that they do not possess a clear IR excess emission typical of very young objects. Their position in the $i-J$ vs. $J-[3.6]$ diagram indicates that their colors, when corrected for the estimated interstellar extinction (Table 4), approach the locus expected for QSOs. Since they are too bright to be high-redshift QSOs, the most likely possibility is that they are heavily reddened low redshift QSOs. Indeed, we estimated that the $\mathrm{H} \alpha$ broad emission line of QSOs could contaminate the magnitude in the methane filters at redfshift $z \sim 1.4$ (Richards et al. 2003).

The SED of ID 3 (Fig. 7) might present IR excess starting from $8 \mu \mathrm{m}$ with a slope $\left(\alpha_{[K \& 24 \mu \mathrm{m}]}=0.27 \pm 0.04\right)$ typical of a class II YSO with a thick disk. However, only flux upper limits are available for $\lambda \geq 5.8 \mu \mathrm{m}$ and, hence, this $\alpha_{[K \& 24 \mu \mathrm{m}]}$ value is not robust. The interpretation of the $K_{S}-$ [3.6] vs. [3.6]-[4.5] CC diagram (Fig. 6) is very uncertain because of the big error bars. However, the position of ID 3 appears to be consistent with the locus of field T-dwarfs. In particular, its [3.6] - [4.5] color is bluer than expected for young mid-T dwarfs such as S Ori 70 and CFHT_J0344+3206. However, assuming that a thick disk surrounds this object, its [3.6] - [4.5] color, peculiar for a young objects, might still be explained in term of different disk inclination with respect to S Ori 70 and CFHT_J0344+3206. Indeed, as shown in the top panels of Fig. 7 by Robitaille et al. (2006), disk inclination significantly alter the SED shape at mid-IR wavelengths $(3-10 \mu \mathrm{m})$ for very low-mass objects $\left(\lesssim 0.2 M_{\odot}\right)$. Finally, its position in the $i-J$ vs. $J-[3.6]$ diagram is clearly consistent with the YSO locus and, in particular, it is very close to the location of S Ori 70. More robust imaging data at mid-IR 
L. Spezzi et al.: Searching for planetary-mass T-dwarfs in the core of Serpens
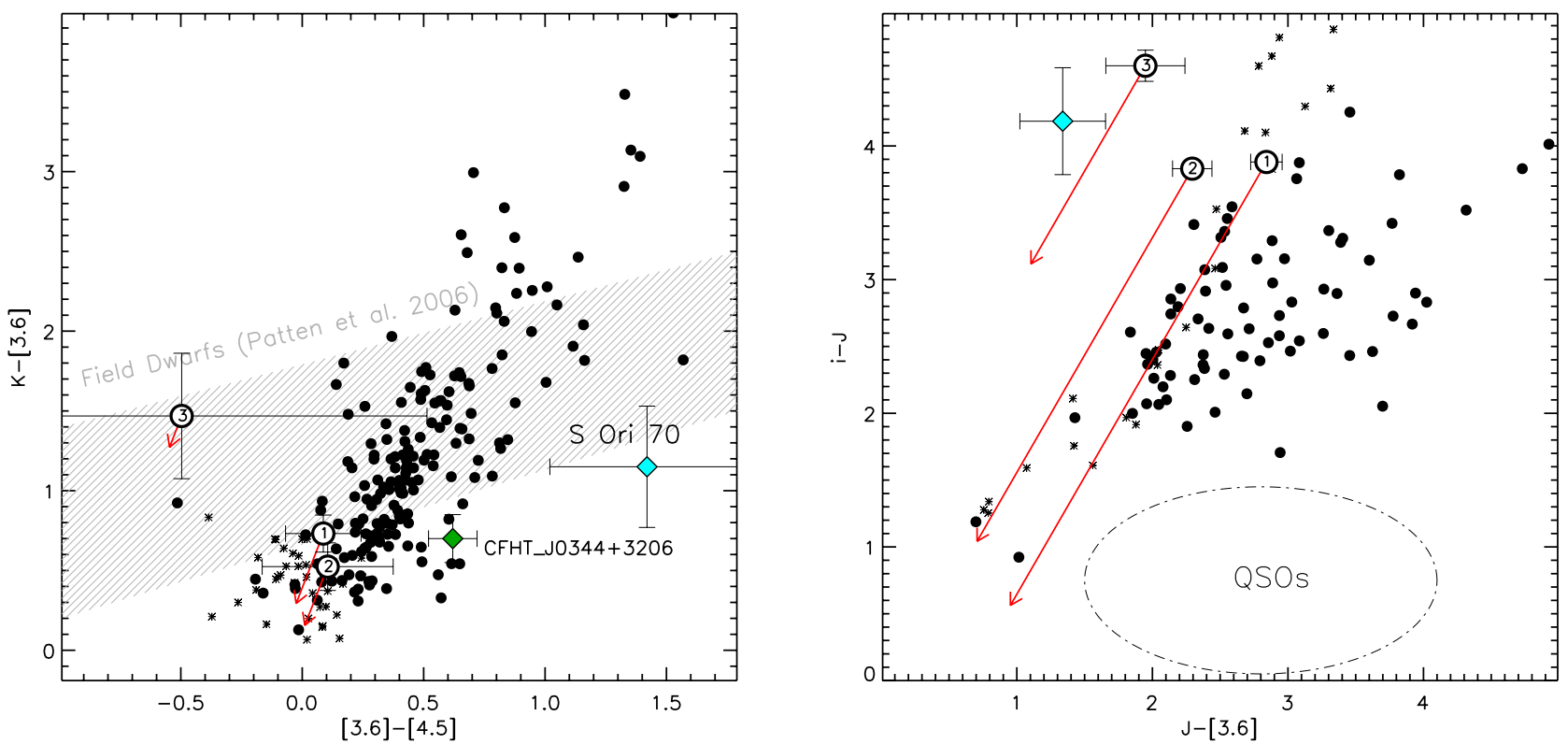

Fig. 6. Left panel: $K_{\mathrm{S}}-[3.6]$ vs. [3.6] - [4.5] color-color diagram. The circles with error bars are our the T-dwarf candidates; their ID number (see Tables 3,4) is indicated and the arrows indicate their position after correction for interstellar reddening. The Serpens Core class II (black dots) and class III (asterisks) YSO population, the S Ori 70 mid-T dwarf (sky-blue diamond), the CFHT_J0344+3206 mid-T dwarf candidate (green diamond) and the field dwarf sequence from Patten et al. (2006) (line-filled area) are plotted for comparison. Righ panel: $i-J$ vs. $J-$ [3.6] color-color diagram. Symbols are as in the left panel. The dot-dashed ellipse marks the area occupied by QSOs in the SWIRE catalog (Hatziminaoglou et al. 2008).
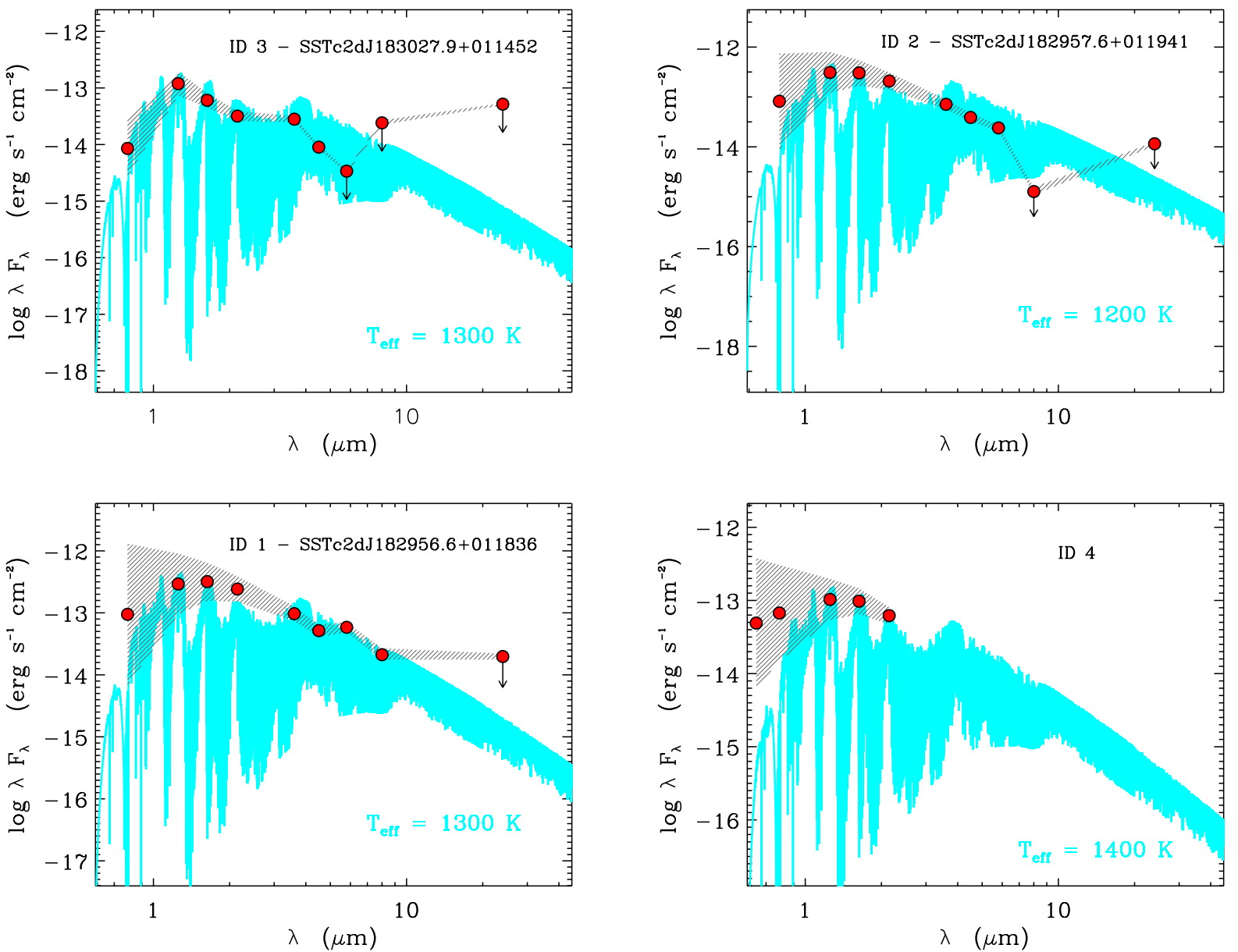

Fig. 7. Spectral energy distributions of the T-dwarf candidates in this study. Flux errors are smaller than the symbol size and flux upper limits are marked with an arrow. The line-filled area represents the flux variation depending on the adopted reddening correction. The oveplotted spectrum is the COND model by Allard et al. (2001) and Baraffe et al. (2003) with the same $T_{\text {eff }}$ as estimated for each object (indicated in the label and in Table 4) normalized to the $J$-band flux. 
wavelengths and/or follow-up spectroscopy is needed to draw a firm conclusion about this object.

ID 4 is not detected in any of the Spitzer pass-bands and, hence, we can not assess the presence of a circumstellar disk around this object. For the sake of completeness, the optical/near-IR SED of ID 4 is shown in Fig. 7. The observed $i-J$ color of ID 4 (i.e., 2.43) is bluer than expected for early/mid T-dwarfs (see, e.g., Table 3 by Hawley et al. 2002), while its observed and dereddened $r-i$ color (1.43 and 0.82 , respectively, adopting the Serpens extinction map $\left(A_{\mathrm{V}}^{\text {map }}\right.$ in Table 4$)$ are consistent with the $r-i$ colors expected for early/mid T-dwarfs (see, e.g., Table 3 by Hawley et al. 2002), in agreement with its near-IR colors (Fig. 5). Thus, our conclusions about this object remain as in Sect. 5.1: ID 4 may be a cluster member, although our data are not sufficient to conclusively prove this and spectroscopic follow-up is required.

\section{Summary and conclusions}

We presented a deep methane imaging survey for planetary-mass T-dwarfs in the Serpens Core cluster. Our survey covers a field of view of $21.5^{\prime} \times 21.5^{\prime}$ and about 127000 sources were detected in both the $\mathrm{CH}_{4}$ on and $\mathrm{CH}_{4}$ off filters.

We identified four potential T-dwarfs from their $1.6 \mu \mathrm{m}$ methane absorption band and used complementary riJHK broad-band imaging and mid-IR flux measurements from the Spitzer c2d Survey to investigate their stellar and disk properties.

Two of our candidates (ID 1 and ID 2) suffer from significant interstellar extinction and have near-IR colors similar to mid-T dwarfs. However, they are too bright to be planetary-mass members of Serpens and do not present the typical IR excess emission expected for young objects surrounded by a disk. Our analysis indicates that their dereddened near-IR colors approach the values expected for QSOs. In particular, since they are too bright to be high-redshift QSOs, they could be heavily reddened low redshift QSOs.

Our candidate ID 3 does not present a significant interstellar extinction and its near-IR colors are more consistent with it being a field mid-T dwarf. It is brighter than field objects at the distance of Serpens and, hence, it may be a foreground T-dwarf. It is detected by the Spitzer c2d survey but only flux upper limits are available for $\lambda \geq 5.8 \mu \mathrm{m}$ and, hence, we can not assess the presence of a possible disk around this object. However, it is worth to keep this possibility open, because the position on the $J$ vs. $J-K_{\mathrm{S}}$ and $i-J$ vs. $J-[3.6]$ diagrams and the estimated spectral type of ID 3 are very similar to those of other young T-dwarf candidates, i.e. S Ori 70 and CFHT_J0344+3206 in the direction of IC 348.

Finally, ID 4 is significantly extincted, which exclude the possibility of it being a foreground object, and has dereddened optical and near-IR colors mainly consistent with those of early/mid T-dwarfs. It is brighter than field objects and, hence, it might be very young, as YSOs have larger radii and are brighter than older field objects of the same spectral class. Thus, ID 4 is a promising young T-dwarf candidate in our sample.

Considering the magnitudes of our candidates ID 3 and 4 (Table 3 ) and their $T_{\text {eff }}$ (Table 4) based on the dereddened $\mathrm{CH}_{4}$ on- $\mathrm{CH}_{4}$ off colors, we estimated their masses assuming that they belong to the Serpens Core cluster (i.e., age $3 \mathrm{Myr}$ and distance in the range 260-415 pc) and using both the COND and DUSTY evolutionary models by Chabrier et al. (2000). The estimated mass is in the range 2-4 Jupiter masses for both ID 3 and 4. Therefore, if they truly belong to the Serpens Core cluster, they would be amongst the youngest, lowest mass objects detected in a star-forming region so far.

Follow-up spectroscopy is required to confirm the spectral type and reddening of our four T-dwarf candidates and, hence, draw firm conclusions about their nature. Additional deep imaging at mid-IR wavelengths is needed to clarify the presence of a possible disk around ID 3 .

Acknowledgements. We acknowledge financial support from IPAG (ANR 2010 JCJC 0501-1) and the Research and Scientific Support Department at ESA-ESTEC. We are grateful to H. Bouy, N. Huelamo and P. Delorme for many discussions during the data analysis, P. Harvey for explanation about the Spitzer c2d catalog and the anonymous referee for his careful reading and useful comments/suggestions. We thank the QSO team at CFHT for their efficient work at the telescope and the data pre-reduction as well as the Terapix group at IAP for the image reduction. This work is based in part on data products produced and image reduction processes conducted at TERAPIX. This research has made use of the NASA/IPAC Infrared Science Archive, which is operated by the Jet Propulsion Laboratory, California Institute of Technology, under contract with the National Aeronautics and Space Administration. This research has also made use of the SIMBAD database, operated at CDS, Strasbourg, France. The research leading to these results has received funding from the European Community's Seventh Framework Programme (/FP7/2007-2013/) under grant agreement No 229517.

\section{References}

Allard, F., Hauschildt, P. H., Alexander, D. R., Tamanai, A., \& Schweitzer, A. 2001, ApJ, 556, 357

Baraffe, I., Chabrier, G., Barman, T. S., Allard, F., \& Hauschildt, P. H. 2003, A\&A, 402, 701

Bertin, E., \& Arnouts, S. 1996, A\&AS, 117, 393

Bertin, E. 2011, Astronomical Data Analysis Software and Systems XX, 442, 435

Boss, A. P. 2001, ApJ, 551, L167

Boulade, O., Charlot, X., Abbon, P., et al. 2003, Proc. SPIE, 4841, 72

Bouy, H., Huélamo, N., Barrado Y Navascués, D., et al. 2009, A\&A, 504, 199

Boyd, D. F. A., \& Whitworth, A. P. 2005, A\&A, 430, 1059

Burgess, A. S. M., Moraux, E., Bouvier, J., et al. 2009, A\&A, 508, 823

Burrows, A., Sudarsky, D., \& Hubeny, I. 2006, ApJ, 640, 1063

Casewell, S. L., Dobbie, P. D., Hodgkin, S. T., et al. 2007, MNRAS, 378, 1131

Casewell, S. L., Jameson, R. F., Burleigh, M. R., et al. 2011, MNRAS, 412, 2071

Chabrier, G. 2003, PASP, 115, 763

Chabrier, G., Baraffe, I., Allard, F., \& Hauschildt, P. 2000, ApJ, 542, 464

Chapman, N. L., Mundy, L. G., Lai, S.-P., \& Evans, N. J. 2009, ApJ, 690, 496

Chiu, K., Fan, X., Leggett, S. K., et al. 2006, AJ, 131, 2722

Davis, C. J., Matthews, H. E., Ray, T. P., Dent, W. R. F., \& Richer, J. S. 1999, MNRAS, 309, 141

Delorme, P., Willott, C. J., Forveille, T., et al. 2008, A\&A, 484, 469

Dobbie, P. D., Kenyon, F., Jameson, R. F., et al. 2002, MNRAS, 335, 687

Dzib, S., Loinard, L., Mioduszewski, A. J., et al. 2010, ApJ, 718, 610

Eiroa, C., Djukvip, A. A., \& Casali, M. M. 2006, Astron. Nachr., 327, 14

Evans, N. J., II, Dunham, M. M., Jörgensen, J. K., et al. 2009, ApJS, 181, 321

Fan, X., Strauss, M. A., Richards, G. T., et al. 2001, AJ, 121, 31

Geballe, T. R., Knapp, G. R., Leggett, S. K., et al. 2002, ApJ, 564, 466

Greene, T. P., Wilking, B. A., Andre, P., Young, E. T., \& Lada, C. J. 1994, ApJ, 434,614

Golimowski, D. A., Leggett, S. K., Marley, M. S., et al. 2004, AJ, 127, 3516

Gorlova, N. I., Meyer, M. R., Rieke, G. H., \& Liebert, J. 2003, ApJ, 593, 1074

Gutermuth, R., Ph.D. Thesis

Harvey, P., Merín, B., Huard, T. L., et al. 2007, ApJ, 663, 1149

Hatziminaoglou, E., Fritz, J., Franceschini, A., et al. 2008, MNRAS, 386, 1252

Hawley, S. L., Covey, K. R., Knapp, G. R., et al. 2002, AJ, 123, 3409

Ichikawa, T., Hamajima, K., Ishida, K., Hidayat, B., \& Raharto, M. 1982, PASJ, 34,231

Jayawardhana, R., Mohanty, S., Basri, G., et al. 2003, Open Issues in Local Star Formation, 299, 251

Kaas, A. A., Olofsson, G., Bontemps, S., et al. 2004, A\&A, 421, 623

Kirkpatrick, J. D., Gelino, C. R., Cushing, M. C., et al. 2012, ApJ, 753, 156

Knapp, G. R., Leggett, S. K., Fan, X., et al. 2004, AJ, 127, 3553

Lada, C. J., \& Wilking, B. A. 1984, ApJ, 287, 610

Leggett, S. K., Saumon, D., Marley, M. S., et al. 2007, ApJ, 655, 1079

Leggett, S. K., Burningham, B., Saumon, D., et al. 2010, ApJ, 710, 1627

Lodieu, N., Caux, E., Monin, J.-L., \& Klotz, A. 2002, A\&A, 383, L15

Lodieu, N., Dobbie, P. D., Deacon, N. R., et al. 2007, MNRAS, 380, 712

Lodieu, N., Hambly, N. C., Dobbie, P. D., et al. 2011, MNRAS, 418, 2604 
Low, C. \& Lynden-Bell, D. 1976, MNRAS, 176, 367

Mainzer, A. K., McLean, I. S., Sievers, J. L., \& Young, E. T. 2004, ApJ, 604, 832

McLean, I. S., McGovern, M. R., Burgasser, A. J., et al. 2003, ApJ, 596, 561

Marmo, C. 2007, in ASP Conf. Ser., 376, 285

Merín, B., Brown, J. M., Oliveira, I., et al. 2010, ApJ, 718, 1200

Miyazaki, S., Komiyama, Y., Sekiguchi, M., et al. 2002, PASJ, 54, 833

Moraux, E., Bouvier, J., Stauffer, J. R., \& Cuillandre, J.-C. 2003, A\&A, 400, 891

Nakajima, T., Oppenheimer, B. R., Kulkarni, S. R., et al. 1995, Nature, 378, 463

Oliveira, I., Merin, B., Pontoppidan, K. M., et al. 2009, ApJ, 691, 672

Patten, B. M., Stauffer, J. R., Burrows, A., et al. 2006, ApJ, 651, 502

Puget, P., Stadler, E., Doyon, R., et al. 2004, in SPIE Conf. Ser. 5492, eds. A. F. M. Moorwood, \& M. Iye, 978

Rebolo, R., Zapatero Osorio, M. R., \& Martín, E. L. 1995, Nature, 377, 129

Rees, M. J. 1976, MNRAS, 176, 483

Richards, G. T., Hall, P. B., Vanden Berk, D. E., et al. 2003, AJ, 126, 1131

Robitaille, T. P., Whitney, B. A., Indebetouw, R., Wood, K., \& Denzmore, P. 2006, ApJS, 167, 256
Santiago, B. X., Gilmore, G., \& Elson, R. A. W. 1996, MNRAS, 281, 871

Schmidt, S. J., West, A. A., Hawley, S. L., \& Pineda, J. S. 2010, AJ, 139, 1808

Scholz, A., Geers, V., Jayawardhana, R., et al. 2009, ApJ, 702, 805

Scholz, A., Muzic, K., Geers, V., et al. 2012, ApJ, 744, 6

Shirono, C., Itho, Y., \& Oasa, Y. 2011, PASJ, 63, 1071

Spezzi, L., Beccari, G., De Marchi, G., et al. 2011, ApJ, 731, 1

Straižys, V., Černis, K., \& Bartašiūtè, S. 2003, A\&A, 405, 585

Testi, L., Sargent, A. I., Olmi, L., \& Onello, J. S. 2000, ApJ, 540, L53

Vrba, F. J., Henden, A. A., Luginbuhl, C. B., et al. 2004, AJ, 127, 2948

Wainscoat, R. J., Cohen, M., Volk, K., Walker, H. J., \& Schwartz, D. E. 1992, ApJS, 83, 111

Weingartner, J. C., \& Draine, B. T. 2001, ApJ, 548, 296

Winston, E., Megeath, S. T., Wolk, S. J., et al. 2007, ApJ, 669, 493

Winston, E., Megeath, S. T., Wolk, S. J., et al. 2009, AJ, 137, 4777

Wright, E. L., Eisenhardt, P. R. M., Mainzer, A. K., et al. 2010, AJ, 140, 1868

Yamauchi, C., Fujishima, S., Ikeda, N., et al. 2011, PASP, 123, 852

Zapatero Osorio, M. R., Béjar, V. J. S., Martín, E. L., et al. 2002, ApJ, 578, 536

Zapatero Osorio, M. R., Béjar, V. J. S., Bihain, G., et al. 2008, A\&A, 477, 895 